Int. J. Morphol.,

30(3):1065-1070, 2012.

\title{
Efecto de un Programa de Estiramientos de la Musculatura Isquiosural en Futbolistas
}

\author{
Effect of Hamstring Stretching Program in Soccer Players
}

\author{
*Raquel Vaquero-Cristóbal; "** José María Muyor; "Fernando Alacid \& ${ }^{* * * *}$ Pedro A. López-Miñarro
}

VAQUERO-CRISTÓBAL, R.; MUYOR, J. M.; ALACID, F. \& LÓPEZ-MIÑARRO, P. A. Efecto de un programa de estiramientos de la musculatura isquiosural en futbolistas. Int. J. Morphol., 30(3):1065-1070, 2012.

RESUMEN: El objetivo fue determinar la influencia de un programa de estiramientos de la musculatura isquiosural en la

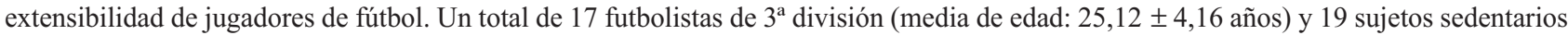
(media de edad: 24,26 \pm 5,64 años) (grupo control) participaron voluntariamente en el estudio. El grupo de futbolistas realizó, durante un periodo de 16 semanas, un plan específico de estiramientos de la musculatura isquiosural, que fue incluido dentro de su plan de entrenamiento, realizando dos series de cuatro estiramientos en las cuatro sesiones semanales de entrenamiento. El grupo control estuvo compuesto por adultos jóvenes varones que no realizaban actividad física alguna. A todos ellos se les valoró la extensibilidad isquiosural al inicio del programa (pre-test), a las 8 semanas (test intermedio) y al finalizar el programa (16 semanas, post-test) mediante el test de elevación de la pierna recta activo (EPR). Se encontró una mejora significativa $(p<0,001)$ en los valores obtenidos por los futbolistas entre el pre-test y el post-test en ambos miembros $\left(68,90 \pm 9,04^{\circ}\right.$ y $77,80 \pm 8,25^{\circ}$ en la pierna derecha; $66,10 \pm 8,15^{\circ}$ y $77,10 \pm 7,77^{\circ}$ en la izquierda, respectivamente). También se encontraron diferencias entre la valoración intermedia y el post-test $(p<0,016)$. Respecto al grupo de sedentarios, los valores del EPR se redujeron paulatinamente entre las tres mediciones. En todas las valoraciones realizadas, los futbolistas alcanzaron valores angulares significativamente superiores respecto a los sujetos sedentarios. La implementación de un programa específico de estiramientos de la musculatura isquiosural, al finalizar las sesiones de entrenamiento, en jugadores de fútbol, produce mejoras en la extensibilidad de esta musculatura, reduciéndose los casos de jugadores con una extensibilidad isquiosural reducida.

PALABRAS CLAVE: Extensibilidad isquiosural; Programa de intervención; Deporte; Flexibilidad.

\section{INTRODUCCIÓN}

Los músculos isquiosurales constituyen un grupo muscular biarticular que, debido a su acción, provoca efectos sobre la rodilla, la cadera, y la dinámica lumbo-pélvica (Ledoux, 1992). Una extensibilidad isquiosural reducida conlleva una mayor cifosis torácica en los movimientos de máxima flexión del tronco (Gajdosik et al., 1994), alteraciones del ritmo lumbo-pélvico (Esola et al., 1996; LópezMiñarro et al., 2012), repercusiones sobre el raquis dorsolumbar (Ferrer, 1998) y lesiones musculares (Cabry \& Shiple, 2000; Croisier et al., 2002; Witvrouw et al., 2003; Henderson et al., 2010).

Diversos estudios han valorado la extensibilidad isquiosural en deportistas, tales como nadadores (Pastor, 2000; Sanz, 2002), corredores de larga distancia (Wang et al., 1993; Trehearn \& Buresh, 2009), piragüistas (GarcíaIbarra et al., 2007; López-Miñarro et al. 2008a, 2008b, 2009b, 2010b; López Miñarro \& Alacid, 2010), remeros (Stuchfield \& Coleman, 2006), halterófilos (Dillon et al., 2004), gimnastas de rítmica (Martínez et al., 2001; Martínez, 2004), futbolistas (Oberg et al., 1984; McIntyre \& Hall, 2005; López-Miñarro et al., 2007a, 2007b; Caldwell \& Peters, 2009; Henderson et al.), jugadores de fútbol australiano (Young et al., 2005), luchadores (Mirzaei et al., 2009), jugadoras de lacrosse (Enemark-Miller et al., 2009), tenistas (Kibler \& Chandler, 2003), taekwondistas (Toskovic et al., 2004), y jugadoras de voleibol (Melrose et al., 2007). La mayoría de estos estudios encuentran una extensibilidad reducida, excepto en aquellos deportes donde la misma tiene un papel muy importante en la ejecución de los gestos técnicos, como en la gimnasia rítmica y la danza.

Otro grupo de estudios ha comparado el grado de extensibilidad isquiosural entre deportistas, tales como

\footnotetext{
* Facultad de Ciencias de la Actividad Física y el Deporte, Universidad Católica San Antonio, Murcia, España.

** Facultad de Educación, Universidad de Almería, Almería, España.

**** Facultad de Educación, Universidad de Murcia, Murcia, España.
} 
halterófilos (Dillon et al.), piragüistas (López-Miñarro et al., 2010a), gimnastas de rítmica (Martínez et al.), corredores de larga distancia (Wang et al.), y jugadoras de élite de lacrosse (Enemark-Miller et al.) con respecto a personas sedentarias, encontrando una mayor extensibilidad en los primeros. No obstante, la mayoría de estos estudios sólo analizan si la práctica de un deporte genera cambios en la extensibilidad isquiosural, sin implementar un programa de intervención.

Son menos los estudios que han evaluado los efectos de un programa de estiramientos de la musculatura isquiosural. Los estudios realizados en tenistas (Kibler \& Chandler), jugadoras de fútbol sala (Ayala et al., 2010), estudiantes universitarios (Ayala \& Sainz de Baranda, 2008), escolares (Sainz de Baranda, 2002, 2009; Zakas et al., 2002; Santonja Medina et al., 2007; Rodríguez-García et al., 2008) y estudiantes de ciclos formativos (Perelló, 2005), han mostrado mejoras significativas en la extensibilidad isquiosural al finalizar la intervención.

Puesto que la reducción de la extensibilidad isquiosural aumenta el riesgo de lesiones (Cabry \& Shiple; Croisier et al.; Witvrouw et al.) y es un factor de riesgo de repercusiones raquídeas, es preciso valorar su extensibilidad y plantear programas de intervención en aquellos casos que sea preciso. Según Arregui \& Martínez de Haro (2001), los entrenamientos específicos realizados habitualmente mejoran la extensibilidad, si bien los entrenamientos genéricos y la competición no logran mejorar esta capacidad. Una inadecuada extensibilidad es, en ocasiones, un problema actitudinal porque los técnicos deportivos y los propios deportistas no consideran la extensibilidad isquiosural como una capacidad importante en la consecución de un alto rendimiento deportivo (Nyland et al., 2004).

Debido a la importancia de una adecuada extensibilidad isquiosural, el objetivo del presente estudio fue determinar la influencia de un programa específico de estiramientos en la extensibilidad isquiosural de futbolistas, así como comparar su evolución respecto a un grupo de sujetos sedentarios.

\section{MATERIAL Y MÉTODO}

Participantes. Un total de 36 sujetos (17 futbolistas que jugaban en la $3^{\text {a }}$ división del fútbol español y 19 sujetos sedentarios) con medias de edad de 25,12 $\pm 4,16$ años y $24,26 \pm 5,64$ años, respectivamente, participaron voluntariamente en este estudio. Los criterios de exclusión fueron haber sido operado de la columna vertebral o musculatura isquiosural, tener alguna alteración raquídea estructurada diagnosticada o algún tipo de lesión en el momento de las valoraciones.

Procedimientos. El estudio fue aprobado por el Comité Ético y de Investigación de la Universidad de Murcia. Previamente a las mediciones, todos los sujetos fueron informados sobre los procedimientos y firmaron, voluntariamente, un consentimiento informado. Además, los participantes cumplimentaron de forma autoadministrada un cuestionario para obtener información sobre su práctica deportiva, volumen de entrenamiento, estiramientos que solían realizar, presencia de dolor raquídeo, frecuencia del mismo y lesiones previas.

El grupo de futbolistas realizó, durante un periodo de 16 semanas, un plan específico de estiramientos estático-activos de la musculatura isquiosural, que fue incluido dentro de su plan de entrenamiento, realizando dos series de cuatro estiramientos en las cuatro sesiones semanales de entrenamiento que realizaban. Los ejercicios de estiramiento se basaron en posturas de cierre del ángulo tronco-muslos en diferentes posiciones (sedentación, bipedestación y decúbito supino), manteniendo el raquis lo más alineado posible. La posición final de estiramiento fue aquella que generaba una sensación de tirantez moderada-intensa en la musculatura isquiosural, pero sin superar el umbral del dolor. La posición de estiramiento se mantuvo durante 20 segundos, con un descanso de 30 segundos entre cada ejercicio. El grupo control no realizó el programa de estiramientos, ni participó en programa alguno de ejercicio físico.

La extensibilidad de la musculatura isquiosural fue evaluada previamente al inicio del programa (pre-test), a las ocho semanas de su comienzo (test-intermedio) y al finalizar el mismo (16 semanas, post-test). Cada sujeto fue evaluado por el mismo examinador en las diferentes mediciones. La temperatura del laboratorio donde se realizaron las mediciones fue estandarizada a $24^{\circ} \mathrm{C}$. Para determinar la extensibilidad isquiosural se utilizó el test de elevación de la pierna recta activo (EPR). Para realizar el test, el sujeto se colocaba en decúbito supino sobre una camilla, con un Lumbosant colocado bajo el raquis lumbar y la pelvis, y realizaba una flexión coxofemoral activa, de forma lenta y progresiva hasta el máximo ángulo que podía alcanzar. La articulación de la rodilla se mantuvo extendida durante el movimiento. La posición del tobillo fue estandarizada en máxima flexión plantar. Un investigador mantuvo la pierna contralateral extendida y en contacto con la camilla, evitando la rotación lateral, así como la rotación de la pelvis en su eje longitudinal. Para determinar el ángulo de flexión coxofemoral se colocó un inclinómetro Unilevel (ISOMED, Inc., Portland, OR) en la tuberosidad tibial, colocándolo a cero grados en la posición inicial y estableciendo los grados 
de flexión coxofemoral al finalizar la misma. La medición se realizó en un orden aleatorio en ambas piernas.

Análisis estadístico. La distribución de los datos fue inicialmente valorada mediante el test de normalidad de Shapiro-Wilks. Puesto que las variables seguían una distribución normal, se realizó un análisis estadístico en base a pruebas paramétricas. Para la obtención de los resultados se realizó una estadística descriptiva con la obtención de los valores medios y desviación típica. Para conocer el efecto del programa de estiramientos en la extensibilidad isquiosural se realizó un análisis de varianza (ANOVA) de dos factores (grupo y test) con medidas repetidas en el segundo factor. La significación del análisis multivariado de medidas repetidas se confirmó mediante los test Traza de Pillai, Lambda de Wilk, traza de Hotelling y raíz mayor de Roy. La esfericidad fue analizada mediante la prueba de Mauchly. La corrección de Greenhouse-Geisser se aplicó si la esfericidad no era asumida. Si se encontraban diferencias significativas en los valores angulares del test EPR para el efecto principal del ANOVA $(p<0,05)$, se procedió a realizar una comparación por pares usando la corrección de Bonferroni para comparaciones múltiples, ajustando el criterio de significación a un valor de $p<0,016$. Para determinar si existían diferencias significativas entre las piernas derecha e izquierda en el test EPR, se realizó una prueba t de Student para muestras dependientes. Todos los datos fueron analizados usando el paquete estadístico SPSS versión 15,0.

\section{RESULTADOS}

El ANOVA reveló diferencias significativas para el efecto principal en los valores del EPR de los futbolistas ( $p$ $<0,001)$. Los valores medios ( \pm desviación típica) del test EPR en el pre-test, la medición intermedia y el post-test, de los futbolistas y grupo control, se presentan en las Tablas I y II, respectivamente. Hubo un aumento significativo ( $p<$ $0,001)$ en los valores angulares del EPR de ambas piernas entre el pre-test y el post-test, así como entre la medición intermedia y el post-test en los futbolistas $(p<0,016)$ (Tabla I). En el grupo control, los valores del EPR fueron disminuyendo, aunque sólo se encontraron diferencias significativas $(p<0,001)$ entre el pre-test y el post-test de la pierna izquierda (Tabla II). Al comparar entre grupos, se detectaron diferencias significativas $(p<0,001)$ en todas las medidas, siendo mayores los valores de los futbolistas en todos los casos. Al comparar los valores angulares entre ambas extremidades, no se encontraron diferencias significativas, ni en los futbolistas ni en el grupo control.

\section{DISCUSIÓN}

El objetivo principal de este estudio fue determinar la influencia de un programa de estiramientos de la musculatura isquiosural en la extensibilidad de este grupo muscular. El hallazgo principal del presente estudio fue una mejora significativa de la extensibilidad isquiosural (de 68,90 \pm $9,04^{\circ}$ a $77,80 \pm 8,25^{\circ}$ en la pierna derecha y de $66,10 \pm 8,15^{\circ}$ a $77,10 \pm 7,77^{\circ}$ en la izquierda) con el trabajo específico de estiramientos, mientras que los sujetos del grupo control mostraron una reducción de los valores angulares del EPR, aunque no significativa. Estos datos concuerdan con estudios previos en deportistas. En jugadoras de fútbol sala se han encontrado mejoras significativas tras un programa de estiramientos, que se reducían rápidamente en las semanas posteriores a la intervención (Ayala et al.). Kibler \& Chandler en jugadores de tenis, encontraron que con un programa de intervención de un año de duración, basado en estiramientos pasivos y activos, se producía una mejora significativa en el test de distancia dedos-planta y en el test EPR, mientras que

Tabla I. Valores medios ( \pm desviación típica) en el test de elevación de la pierna recta del grupo de intervención (futbolistas).

\begin{tabular}{lccc}
\hline & EPR pre-test & EPR test-intermedio & EPR post-test \\
\hline Pierna derecha & $68,90 \pm 9,04^{\circ}$ & $72,10 \pm 6,12^{\circ}$ & $77,80 \pm 8,25^{\circ} * \dagger$ \\
Pierna izquierda & $66,10 \pm 8,15^{\circ}$ & $72,90 \pm 6,22^{\circ}$ & $77,10 \pm 7,77^{\circ} * \dagger$ \\
\hline
\end{tabular}

EPR: test de elevación de la pierna recta activo; ${ }^{*} p<0,001$ respecto al pre-test; $\uparrow p<0,016$ respecto al testintermedio.

Tabla II. Valores medios ( \pm desviación típica) en el test de elevación de la pierna recta del grupo control (sedentarios).

\begin{tabular}{lccc}
\hline & EPR pre-test & EPR test-intermedio & EPR post-test \\
\hline Pierna derecha & $60,57 \pm 11,77^{\circ}$ & $59,15 \pm 10,24^{\circ}$ & $57,94 \pm 11,46^{\circ}$ \\
Pierna izquierda & $60,73 \pm 10,23^{\circ}$ & $58,63 \pm 10,31^{\circ}$ & $55,57 \pm 11,76^{\circ} *$ \\
\hline
\end{tabular}

EPR: test de elevación de la pierna recta activo; $* p<0,001$ respecto al pre-test. 
el grupo control empeoraba sus valores. Otros estudios han valorado el efecto de un programa de estiramientos en ámbito escolar. Zakas et al. tras un programa de doce semanas, realizando estiramientos de la musculatura isquiosural tres veces por semana, mostraron un mayor rango de movimiento $(p<0,001)$ respecto a un grupo control, en todos los rangos de edad. Otros estudios de mayor duración (9 meses, dos sesiones semanales, 5 minutos de estiramientos de la musculatura isquiosural), en escolares de primaria y secundaria, que realizaban estiramientos en sus clases obligatorias de Educación Física, han mostrado mejoras significativas en los test EPR y distancia dedos-planta, siendo mayores cuanto más volumen de estiramientos se realizaba (Santonja Medina et al.; Rodríguez-García et al.).

Los valores obtenidos por los deportistas, en el test EPR, fueron significativamente mayores que los alcanzados por los sujetos sedentarios en todas las mediciones realizadas. La mayor extensibilidad isquiosural de los futbolistas en el pre-test se explican por el hecho de que estos estiraban de forma aislada y con un volumen reducido antes de sus entrenamientos. Arregui \& Martínez de Haro, en un trabajo de revisión bibliográfica, establecieron que los entrenamientos específicos realizados habitualmente mejoran la extensibilidad, si bien los entrenamientos genéricos y la competición no logran mejorar esta capacidad. Por este motivo, se ha descrito con mucha frecuencia un alto porcentaje de deportistas con una extensibilidad isquiosural reducida (Oberg et al.; Pastor; Sanz; McIntyre \& Hall; Young et al.; Stuchfield \& Coleman; López-Miñarro et al., 2007a, 2007b, 2010b; Caldwell \& Peters; Trehearn \& Buresh; López Miñarro \& Alacid). Diversos estudios en piragüistas han encontrado un gran predominio de sujetos con una baja extensibilidad y un alto porcentaje de casos de cortedad grado I, especialmente en canoístas (García-Ibarra et al.; López-Miñarro et al., 2008a, 2008b, 2010b, 2011), y se justifica por la au- sencia de un trabajo específico de esta musculatura debido a la menor influencia que la extensibilidad isquiosural tiene en su rendimiento deportivo. En otro estudio, LópezMiñarro et al. (2009a) encontraron una mayor extensibilidad isquiosural en corredores que en piragüistas, debido a que los primeros suelen realizar estiramientos por la mayor implicación del tren inferior en su práctica. En este sentido, algunos estudios comparan la extensibilidad de deportistas con sujetos sedentarios, pero sin realizar intervención alguna. Dillon et al. encontraron que los deportistas de halterofilia obtenían mayores distancias en el test de distancia dedos-planta que sujetos sedentarios de la misma edad. Por su parte, Wang et al. encontraron que atletas de larga distancia conseguían mayores valores angulares en el test EPR que personas sedentarias.

Una extensibilidad isquiosural reducida conlleva una mayor cifosis torácica en los movimientos de máxima flexión del tronco (Gajdosik et al.), alteraciones del ritmo lumbopélvico (Esola et al.; López-Miñarro et al., 2012), repercusiones sobre el raquis dorso-lumbar (Ferrer; Muyor et al., 2011) y lesiones musculares (Cabry \& Shiple; Croisier $e t$ al.; Witvrouw et al.; Henderson et al.). En esta línea, un reciente estudio en futbolistas profesionales encontró que un rango de flexión activa de cadera inferior al normal aumentaba las posibilidades de sufrir una lesión en la musculatura isquiosural (Henderson et al.). Por todo ello, es preciso incluir programas de estiramiento isquiosural en los entrenamientos de los futbolistas.

En conclusión, la implementación de un programa específico de estiramientos de la musculatura isquiosural en jugadores de fútbol, basado en estiramientos estático-activos manteniendo el raquis alineado, realizados al finalizar las sesiones de entrenamiento, produce mejoras en la extensibilidad de esta musculatura, reduciéndose los casos de jugadores con cortedad de la musculatura isquiosural.

VAQUERO-CRISTÓBAL, R.; MUYOR, J. M.; ALACID, F. \& LÓPEZ-MIÑARRO, P. A. Effect of hamstring stretching program in soccer players. Int. J. Morphol., 30(3):1065-1070, 2012.

SUMMARY: The objective was to determine the influence of hamstring stretching program on hamstring extensibility in soccer players. Seventeen amateur soccer players (mean age: $25.12 \pm 4.16$ years) and 19 sedentary subjects (control group) (mean age: $24.26 \pm$ 5.64 years) were recruited. The soccer players group performed a hamstring stretching program during the four weekly training sessions for a period of 16 weeks. The stretching program included four exercises and each session consisted of 2 sets of each exercise. The control group did not participate in any hamstring-stretching or physical exercise program. Hamstring muscle extensibility was determined by active straight leg raise angle before (pre-test), at 8 weeks (intermediate-test) and after the 16 weeks (post-test) hamstring-stretching program. Significant improvements $(\mathrm{p}<0.001)$ were found in SLR angles for the experimental group in post-test for both limbs $(68.90$ $\pm 9.04^{\circ}$ and $77.80 \pm 8.25^{\circ}$ in the right leg; $66.10 \pm 8.15^{\circ}$ and $77.10 \pm 7.77^{\circ}$ in the left leg, respectively). The SLR values decreased between measures in sedentary subjects. Active straight leg raise values of the soccer players were significantly higher in all measures. This study indicates that a stretching program performed at finish of training sessions increases the hamstring extensibility in soccer players, reducing the frequency of players with reduced hamstring extensibility.

KEY WORDS: Hamstring extensibility; Intervention program; Flexibility; Sport. 


\section{REFERENCIAS BIBLIOGRÁFICAS}

Arregui, J. A. \& Martínez de Haro, V. Estado actual de las investigaciones sobre la flexibilidad en la adolescencia. Rev. Int. Med. Cienc. Act. Fís. Deporte, 1(2):127-35, 2001.

Ayala, F. \& Sainz de Baranda, P. Efecto del estiramiento activo sobre el rango de movimiento de la flexión de cadera: 15 versus 30. Motricidad, 20:2-14, 2008.

Ayala, F.; Sainz de Baranda, P.; Cejudo, A. \& De Ste Croix, M. Efecto de un programa de estiramientos activos en jugadoras de fútbol sala de alto rendimiento. Cult. Cienc. Deporte, 5(15):159-67, 2010.

Cabry, J. \& Shiple, B. J. Increasing hamstring flexibility decreases hamstring injuries in high school athletes. Clin. J. Sport. Med., 10(4):311-2, 2000.

Caldwell, B. P. \& Peters, D. M. Seasonal variation in physiological fitness of a semiprofessional soccer team. J. Strength Cond. Res., 23(5):1370-7, 2009.

Croisier, J. L.; Forthomme, B.; Namurois, M. H.; Vanderthommen, M. \& Crielaard, J. M. Hamstring muscle strain recurrence and strength performance disorders. Am. J. Sports Med., 30(2):199-203, 2002.

Dillon, C.; Paulose-Ram, R.; Hirsch, R. \& Gu, Q. Skeletal muscle relaxant use in the United States: data from the Third National Health and Nutrition Examination Survey (NHANES III). Spine (Phila Pa 1976), 29(8):892-6, 2004.

Enemark-Miller, E. A.; Seegmiller, J. G. \& Rana, S. R. Physiological profile of women's Lacrosse players. J. Strength Cond. Res., 23(1):39-43, 2009.

Esola, M. A.; McClure, P. W.; Fitzgerald, G. K. \& Siegler, S. Analysis of lumbar spine and hip motion during forward bending in subjects with and without a history of low back pain. Spine (Phila Pa 1976), 21(1):71-8, 1996.

Ferrer, V. Repercusiones de la cortedad isquiosural sobre la pelvis y el raquis lumbar. Tesis Doctoral. Murcia, Universidad de Murcia, 1998.

Gajdosik, R. L.; Albert, C. R. \& Mitman, J. J. Influence of hamstring length on the standing position and flexion range of motion of the pelvic angle, lumbar angle, and thoracic angle. J. Orthop. Sports Phys. Ther., 20(4):213-9, 1994.

García-Ibarra, A.; López-Miñarro, P. A.; Alacid, F.; Ferragut, C. \& Yuste, J. L. Comparación de la extensibilidad isquiosural y la flexión del raquis lumbar entre canoistas y kayakistas de categoría infantil. III Congreso Internacional de Ciencias del Deporte, 29-31 Marzo, Pontevedra, España, 2007.
Henderson, G.; Barnes, C. A. \& Portas, M. D. Factors associated with increased propensity for hamstring injury in English Premier League soccer players. J. Sci. Med. Sport., 13(4):397402, 2010.

Kibler, W. B. \& Chandler, T. J. Range of motion in junior tennis players participating in an injury risk modification program. J. Sci. Med. Sport, 6(1):51-62, 2003.

Ledoux, P. L'extensibilité des ischio-jambiers. Kinésithérapie Scientifique, 313:6-8, 1992.

López-Miñarro, P. A. \& Alacid, F. Influence of hamstring muscle extensibility on spinal curvatures in young athletes. Science \& Sports, 25(4):188-93, 2010.

López-Miñarro, P. A.; Alacid, F.; Ferragut, C. \& García, A. Valoración y comparación de la disposición sagital del raquis entre canoístas y kayakistas de categoría infantil. Cult. Cienc. Deporte, 9:171-6, 2008a.

López-Miñarro, P. A.; Alacid, F.; Ferragut, C. \& García, A. Valoración y comparación de la extensibilidad isquiosural entre kayakistas y canoistas de categoría infantil. Motricidad, 20:97-111, 2008b.

López-Miñarro, P. A.; Alacid, F. \& Muyor, J. M. Comparación del morfotipo raquídeo y extensibilidad isquiosural entre piragüistas y corredores. Rev. Int. Med. Cienc. Act. Fís. Deporte, 9(36):379-92, 2009a.

López-Miñarro, P. A.; Alacid, F. \& Rodríguez-García, P. L. Comparison of sagittal spinal curvatures and hamstring muscle extensibility among young elite paddlers and nonathletes. Int. Sportmed. J., 11(2):301-12, 2010a.

López-Miñarro, P. A.; Muyor, J. M. \& Alacid, F. Sagittal spinal curvatures and pelvic tilt in elite young kayakers. Med. Sport. 63(4):509-19, 2010 b.

López-Miñarro, P. A.; Muyor, J. M. \& Alacid, F. Influence of hamstring extensibility on sagittal spinal curvatures and pelvic tilt in highly trained young kayakers. Eur. J. Sport. Sci., 12(6):469-74, 2012.

López-Miñarro, P. A.; Rodríguez, P. L.; Santonja, F.; Yuste, J. L. \& García, A. Sagittal spinal curvatures in recreational weight lifters. Arch. Med. Deporte, 122:435-441, 2007a.

López-Miñarro, P. A.; Yuste, J. L.; Rodríguez, P. L.; Santonja, F.; Sáinz de Baranda, P. \& García, A. Sagittal spinal curvatures of thoracic and lumbar spine during the standing bilateral curl bar exercise. Cult. Cienc. Deporte, 7(3):19-24, 2007 b.

Martínez, F. M. Disposición del raquis en el plano sagital y 
extensibilidad isquiosural en gimnasia rítmica deportiva. Tesis Doctoral, Murcia, Universidad de Murcia, 2004.

Martínez, F.; Pastor, A. \& Rodríguez, P. L. Estudio del morfotipo sagital de la columna y de la extensibilidad de la musculatura isquiosural en gimnasia rítmica deportiva. En: Díaz, A. \& Segarra, E. Actas del $2^{\circ}$ Congreso Internacional de Educación Física y Diversidad. Madrid, Gymnos, 2001. pp.33048.

McIntyre, M. C. \& Hall, M. Physiological profile in relation to playing position of elite college Gaelic footballers. Br. J. Sports Med., 39(5):264-6, 2005.

Melrose, D. R.; Spaniol, F. J.; Bohling, M. E. \& Bonnette, R. A. Physiological and performance characteristics of adolescent club volleyball players. J. Strength Cond. Res., 21(2):481-6, 2007.

Mirzaei, B.; Curby, D. G.; Rahmani-Nia, F. \& Moghadasi, M. Physiological profile of elite Iranian junior freestyle wrestlers. J. Strength Cond. Res., 23(8):2339-44, 2009.

Muyor, J. M.; Alacid, F. \& López-Miñarro, P. A. Sagittal spinal morphology in young highly-trained paddlers. Int. J. Morphol., 29(3):1047-53, 2011.

Nyland, J.; Kocabey, Y. \& Caborn, D. N. Sex differences in perceived importance of hamstring stretching among high school athletes. Percept. Mot. Skills, 99(1):3-11, 2004.

Oberg, B.; Ekstrand, J.; Möller, M. \& Gillquist, J. Muscle strength and flexibility in different positions of soccer players. Int. J. Sports Med., 5(4):213-6, 1984.

Pastor, A. Estudio del morfotipo sagital de la columna y de la extensibilidad de la musculatura isquiosural de jóvenes nadadores de élite Españoles. Tesis Doctoral, Murcia, Universidad de Murcia, 2000.

Perelló, V. Estudio de la musculatura de la región posterior del muslo tras programa de estiramientos. Tesis Doctoral, Valencia, Universidad de Valencia, 2005.

Rodríguez-García, P. L; López-Miñarro, P. A.; Yuste, J. L. \& Sáinz de Baranda, P. Comparison of hamstring criterion-related validity, sagittal spinal curvatures, pelvic tilt, and score between sit-and-reach and toe-touch tests in athletes. Medicina dello Sport, 61(1):11-20, 2008.

Sainz de Baranda, P. Educación física y actividad extraescolar: programa para la mejora del raquis en el plano sagital y la extensibilidad isquiosural en Primaria. Tesis Doctoral, Murcia, Universidad de Murcia, 2002.

Sainz de Baranda, P. El trabajo de la flexibilidad en educación física. Programa de intervención. Cult. Cienc. Deporte, 10:338, 2009.
Santonja Medina, F. M.; Sainz De Baranda Andújar, P.; Rodríguez García, P. L.; López Miñarro, P. A. \& Canteras Jordana, M. Effects of frequency of static stretching on straight-leg raise in elementary school children. J. Sports Med. Phys. Fitness, 47(3):304-8, 2007.

Sanz, I. Natación y flexibilidad. Rev. Int. Med. Cienc. Act. Fís. Deporte, 2(6):128-42, 2002.

Stutchfield, B. \& Coleman, S. The relationships between hamstring flexibility, lumbar flexion, and low back pain in rowers. Eur. J. Sports Sci., 6(4):255-60, 2006.

Toskovic, N. N.; Blessing, D. \& Williford, H. N. Physiologic profile of recreational male and female novice and experienced Tae Kwon Do practitioners. J. Sports Med. Phys. Fitness, 44(2):164-72, 2004.

Trehearn, T. L. \& Buresh, R. J. Sit-and-reach flexibility and running economy of men and women collegiate distance runners. J. Strength Cond. Res., 23(1):158-62, 2009.

Wang, S. S.; Whitney, S. L.; Burdett, R. G. \& Janosky, J. E. Lower extremity muscular flexibility in long distance runners. J. Orthop. Sports Phys. Ther., 17(2):102-7, 1993.

Witvrouw, E.; Danneels, L.; Asselman, P.; D'Have, T. \& Cambier, D. Muscle flexibility as a risk factor for developing muscle injuries in male professional soccer players. A prospective study. Am. J. Sports Med., 31(1):41-6, 2003.

Young, W. B.; Newton, R. U.; Doyle, T. L.; Chapman, D.; Cormack, S.; Stewart, G. \& Dawson, B. Physiological and anthropometric characteristics of starters and non-starters and playing positions in elite Australian Rules Football: a case study. J. Sci. Med. Sport, 8(3):333-45, 2005.

Zakas, A.; Galazoulas, C.; Grammatikopoulou, M. G. \& Vergou, A. Effects of stretching exercise during strength training in prepubertal, pubertal and adolescent boys. J. Bodyw. Mov. Ther., 6(3):170-6, 2002.

\author{
Dirección para correspondencia: \\ Fernando Alacid \\ Facultad de Ciencias de la Actividad Física y el Deporte \\ Universidad Católica San Antonio \\ Campus de los Jerónimos s/n \\ 30107 - Guadalupe \\ Murcia \\ SPAIN
}

Email: raquel.vaquero@um.es

Recibido : 08-11-2011

Aceptado: 11-06-2012 